\title{
La representación del mundo en la Edad Media: la cartografía
}

\author{
Alonso Ríos GonZÁLEZ \\ Universidad Nacional Autónoma de México
}

\begin{abstract}
El presente texto tiene por finalidad repasar de manera breve los principales aspectos de la cartografía medieval y particularmente de los mapas del mundo realizados en aquella época. Entre los principales aspectos a notar de estas representaciones del mundo se encuentra su desatención a las relaciones de distancia y escala, así como a la naturaleza funcional de la cartografía, a las cuales se nos ha acostumbrado a partir del surgimiento del cartesianismo. En cambio, los mappaemundi medievales parecen responder eficazmente a la definición de "mapa" propuesta por Peter Jackson, para quien este concepto engloba toda representación gráfica de conocimiento. Finalmente, la lectura que se propone de los mapas del mundo medieval se lleva a cabo desde el tamiz de las teorizaciones sobre el espacio de la escuela lefebvriana y en particular a partir del papel que el filósofo francés da a las representaciones dentro del proceso de la producción del espacio social.
\end{abstract}

PALABRAS CLAVE: cartografía medieval, espacio, mappaemundi, representaciones del mundo.

This essay intends to make a short review of the main aspects of medieval cartography, especially of the maps of the world made during this period. Among the main characteristics to observe in these forms of representations of the world, one can find the lack of attention to distance and scale accuracy, as well as to the functional nature of cartography imposed by Cartesian thinking. To the contrary, medieval mappaemundi seem to efficiently exemplify the "map" definition given by Peter Jackson for whom this concept includes every graphic representation of knowledge. Finally, the reading here proposed about medieval world maps is made under the perspective of lefebvrian space theorizations, specially from the point of view proposed by the French philosopher about the role of representation in the process of the production of social space.

KEYWORDS: medieval cartography, space, mappaemundi, world representations.

En el campo de los estudios literarios y del arte, nos resulta claro entender al espacio como una especie de "ilusión" generada por medios discursivos que responde más o menos al intento de "representar" un mundo de acción humana: los lugares donde ocurre una historia son esencialmente intangibles pues existen sólo como discurso, aunque, sobre todo en las ficciones de corte realista, son también percibidos como representaciones del "espacio real". 


\section{$16 \square$ LA REPRESENTACIÓN DEL MUNDO EN LA EDAD MEDIA: LA CARTOGRAFÍA}

Desde otras perspectivas, ese espacio real lo entendemos como aquel que se puede cuantificar, transitar, ocupar y habitar. Sin embargo, a partir de la última parte del siglo XX, en las ciencias sociales ha tenido eco una tendencia teórica que pone en duda la efectiva materialidad de este concepto y, por lo tanto, la verdadera posibilidad de un “espacio absoluto". En fin, en gran parte gracias a la propuesta de Henri Lefebvre en torno a la producción del espacio social, se ha comenzado a pensarlo, también, como una suerte de fenómeno discursivo que encuentra su realización sólo a partir de las prácticas y de las representaciones que en él y sobre él se llevan a cabo. Hubbard explica que "Lefebvre infers that absolute space cannot exist because, at the moment it is colonised through social activity, it becomes relativised and historicised space" (2005: 42), y por su parte Rober Tally dice: "Lefebvre's astonishingly bold thesis is already announced in the title. Space, here meaning social space, is not the empty container of Cartesian or Kantian thought, but a social product made possible by human effort" (2013: 117).

El espacio así entendido depende en gran medida, para su realización, de la forma en que se le concibe y se le representa; y a propósito afirma que las representaciones del espacio, "es decir, el espacio concebido, el espacio de los científicos, planificadores, urbanistas, tecnócratas fragmentadores, ingenieros sociales y hasta el de cierto tipo de artistas próximos a la cientificidad, todos los cuales identifican lo vivido y lo percibido con lo concebido [...] es el espacio dominante en cualquier sociedad" (Lefevre, 2013: 97). Su importancia radica en que tales representaciones tendrían "un impacto considerable y una influencia específica en la producción del espacio" (100), y continúa: "Quizás sea preciso ir más allá y admitir que los productores del espacio han actuado siempre de acuerdo a una representación, mientras que los 'usuarios' han experimentado pasivamente lo que les ha sido impuesto, más o menos insertado o justificado en su espacio de representación" (2013: 102). En otras palabras, se puede decir que la forma en que el espacio es representado acaba por determinar las prácticas que en él se han de llevar a cabo.

Siguiendo esta escuela de pensamiento, hacia finales del siglo XX Peter Jackson realiza una crítica deconstructiva en aquello que para Ola Sönderström es el "geography's tool of representation 'par excellence': cartography" (2005: 13). La perspectiva de Jackson se centra en las cualidades significativas del mapa, y supone la premisa, ya avanzada por Bryan Harley, de que "all maps are cultural artefacts and, as tools of those with wealth and authority, are inescapably bound as ideological instruments into the nexus of power-knowledge" (Cosgrove, 2005: 29). Me interesa subrayar aquí la consideración de que "The Western mapping tradition, with its focus on rational, geometrically based spatial scaling, classification and allocation is thus revealed as merely one, culturally specific, mode of geographical representation rather than a timeless and universal technique of graphic communication" (29). En otras palabras, los mapas, como medio privilegiado de la representación del espacio, muestran y ocultan aspectos de la realidad según las exigencias de los contextos y los agentes que los producen. 


\section{Los mapas del mundo en la época medieval}

Entre los autores que se dedican a la cartografía medieval, una afirmación parece sostenerse con regularidad: que "Durante la Edad Media lo que llamamos mapa era en esencia una pintura" (Torres, 2013: 7). Sergio Octavio Torres, de hecho, en un punto se pregunta si debería incluirse dentro de la cartografía (un concepto decididamente geográfico) a los mapas del mundo medievales cuyas principales funciones representacionales no eran de carácter geográfico. Por su parte, Mattheu Ducza nos recuerda que "in Medieval times, neither church Latin nor vernacular languages contained an equivalent for our modern word "map"' (2013: 8). Lo que para dicha época es considerado como mapas, y en particular como mapas del mundo o mappaemundi, se relaciona con la cartografía desarrollada desde el Renacimiento hasta nuestros días, en tanto que todos estos ejemplos entran en la definición de Peter Jackson que entiende al mapa como "all graphic representations of knowledge" (Cosgrove, 2005: 28).

A este respecto es muy claro cómo la cartografía medieval tiene por objetivo representar gráficamente el conocimiento de su época. Pero antes de ahondar en ello conviene delinear brevemente el complejo panorama de lo que hoy se entiende por cartografía medieval. A propósito de esto, P. D. A. Harvey advierte cómo no había, en el Medioevo, una idea general de mapa, y que cada una de las representaciones que hoy consideramos dentro de este conjunto era elaborada con un propósito particular, influido quizá por el texto al que acompañaba o que la acompañaba y en mucha menor medida por sus contrapartes gráficas. En este contexto es comprensible que una clasificación unívoca de la cartografía medieval resulte impracticable, sin embargo, algunas generalidades se han podido establecer.

En primer lugar, Harvey distingue "several quite distinct traditions of medieval maps, notably the mappaemundi, the portolan charts, the regional and local maps, and a relatively small corpus of celestial maps" (1987b: 283), tradiciones que no se sustituyen entre sí, sino que más bien conviven durante prolongados periodos del Medioevo. Las cartas portulanas, por su parte, cuyo origen no ha sido claramente dilucidado, son una especie de mapas náuticos que surgieron hacia mediados del siglo XIII y tuvieron vigencia hasta el siglo XVI. Su propósito era cartografiar los bordes del Mediterráneo, señalando los puertos y los accidentes litorales (lo cual se lograba de manera aproximada) con la finalidad de marcar ciertos rumbos de navegación en dicho mar. Por su parte, los mapas regionales son así llamados precisamente porque se caracterizan por representar sólo determinada región (villas, pueblos, ciudades y en raras ocasiones países enteros). Pese a que este corpus es tan escaso como diverso, Harvey distingue determinadas características más o menos generales entre sus elementos:

Whether they show a large area or a small one, they are all conceived as showing it from above, either vertically or obliquely, viewed from a position often unattainable in reality; this has been taken as defininga map for our purpose. These are the products of medieval Europe that are typologically, historically, or conceptually 


\section{$18 \square$ LA REPRESENTACIÓN DEL MUNDO EN LA EDAD MEDIA: LA CARTOGRAFÍA}

the precursors of the large-scale and topographic maps of the sixteenth century and later (1987a: 464).

Los mappaemundi, por su parte, son representaciones del mundo entero con todo lo que ello implica para el mundo medieval. Como bien se sabe, el universo en la época es concebido como geocéntrico, esférico, finito y eterno; y se halla dividido entre el mundo sublunar y el supralunar, según la adaptación cristianizada del modelo ptolemaico-aristotélico. Por lo tanto, la primera nota distintiva de estas formas cartográficas es que "De esta imagen del universo, los mappaemundi medievales deben de ser entendidos como la proyección de la esfera más interna, es decir, como la imagen de la Tierra" (Sáenz-López, 2004: 30). Por otro lado, la representación del mundo así realizada no se ciñe a meras coordenadas espaciales sino que implica, siguiendo el espíritu enciclopédico de la época, muchos más aspectos: "Los mapas de la Edad Media no sólo reproducen el mundo en cuanto a su acepción física, es decir, la Tierra; los numerosos textos e ilustraciones que incorporan lo muestran desde una definición más amplia — enciclopédica — que integra también cuestiones científicas, históricas y religiosas" (14).

Para un lector contemporáneo, la lectura de este tipo de mapas puede resultar complicada, pues no se remite en momento alguno a la descripción matemática del espacio físico, como se nos ha acostumbrado desde el Renacimiento. Para leerlos correctamente, entonces, es necesario asimilar el pensamiento medieval y reconocer, en primer lugar, que lo que importaba representar no era la mímesis del mundo observable, sino la concepción plena del mundo en la época, en sus aspectos físicos, pero sobre todo en aquellos filosóficos, morales, históricos, religiosos, etcétera. Por ello, más que la representación mimética, va a resultar de particular relevancia la representación simbólica de las cosas terrenas: "They presented a network of information, detailing not only geographical features but also zoological, anthropological, moral, theological and historical ideas" (Ducza, 2013: 8).

En este punto conviene recordar que, en el ámbito intelectual de la Edad Media, la adopción del modelo ptolemaico-aristotélico implicaba empatar el pensamiento filosófico clásico con la doctrina bíblica, así como con la experiencia física, lo cual implicó un álgido debate entre los intelectuales del periodo. Uno de los cuestionamientos más interesantes era decidir el modo en que la tierra podía ocupar la esfera central del universo y aún así tener una porción elevada por encima del agua de modo que la vida fuera posible. Según nos informa Julia Benavent: "algunos hablaban de la excentricidad entre los elementos, de sus respectivas esferas" (2016: 23), mientras otros "decían que había una concentricidad plena de la tierra y del agua, y [...] para ellos la tierra había emergido por voluntad de Dios" (23-24). A esta última corriente se adscribe el mismo Dante Alighieri cuando en su Questio de Aqua et Terra concluye: "aunque la tierra, según su simple natura, tiende igualmente al centro [...], sin embargo, según otra naturaleza, que sigue a la naturaleza universal, se deja elevar dócilmente en una parte para hacer posible la mezcla de los elementos" (46-47). Como sea, una de las repre- 
sentaciones más aceptadas del mundo en la época es que la tierra tenía un hemisferio de tierras elevadas y uno de sólo agua.

Lo interesante aquí es que tales discusiones, hipótesis y resoluciones, hallaron su contraparte en la expresión gráfica de los mappaemundi. De tal modo que el margen de agua circular que se ha mencionado antes en muchos casos estaba en representación del entero hemisferio austral. Más aún, Sáenz-López propone que: "Frente a la aparente planitud del mundo en las representaciones cartográfcas, ciertos detalles geográfcos de algunos mappaemundi medievales han sido interpretados como intentos de aludir a la esfericidad terrestre" (2016: 31). Un ejemplo interesante es la ilustración que se halla en el manuscrito del siglo XIV del Breviario de amor de Matfre Ermengaud (ver Sáenz-López, 2016: 31), en el que el Océano es representado como un cascarón de huevo partido por la mitad del que emerge, por la parte superior, la mitad de la esfera terrestre.

En otros ejemplos inclusive la esfera del aire, representada por medio de los vientos, ocupaba los márgenes de la ilustración, es decir, más allá de las aguas, como sería su adecuada ubicación. Según nos dice Sáenz-López: “Algunos mapas medievales dejan entrever su imbricación cósmica al disponer alrededor del mapa (que aúna en sí la Tierra y la esfera de agua a través del océano circundante), los vientos, alusivos a la esfera de aire. Incluso el mappamundi de Ripoll incluye una corona de fuego que cierra el mundo sublunar" (2016: 32). ${ }^{1}$

Otra característica formal importante de los mappaemundi es la disposición tripartita de la tierra, cuya representación más común es la llamada T-O, pues al interior de una circunferencia que marca el lugar de la esfera del agua, se organizan, en forma de T, los tres continentes conocidos al momento (Asia, Europa y África, que son divididos por los principales cuerpos de agua como el mar Mediterráneo y el río Nilo: "Esta tripartición del mundo arraigó con gran fuerza en el Medievo, pues la descripción geográfica coincidía con los textos sagrados que explicaban que, tras el Diluvio universal, Noé repartió la tierra entre sus tres hijos" (Sáenz-López, 2016: 34). En estos mapas, Asia, en cuyo extremo habría de encontrarse el Paraíso, solía ser ubicada en la parte superior del plano, de este modo: "Following the circumference of a mappamun$d i$ from the western point at the bottom, to the south and then east, the viewer moves from the normal world, through the unknown and finally arrives at Paradise. To move farther southwards was to go farther from God" (Ducza, 2013: 13). Como Matthew Ducza apunta, concebir un Paraíso localizable en la tierra da cuenta de la creencia de un Dios que tiene una presencia efectiva en el espacio físico.

Otro aspecto interesante, que igualmente puede resultar chocante al lector contemporáneo, es que en estos mappaemundi no había un respeto sistemático por las relacio-

\footnotetext{
${ }^{1}$ Por su mayor ligereza, en el modelo aristotélico, el fuego debería ocupar la más alta esfera del mundo sublunar. La lógica medieval aceptaba esta verdad, aunque no pudiera ser verificada empíricamente. Por ejemplo, dentro de la Divina commedia, Dante y Beatriz superan esta esfera antes de ascender al cielo de la luna (Par. I, 97-141).
} 


\section{$20 \square$ LA REPRESENTACIÓN DEL MUNDO EN LA EDAD MEDIA: LA CARTOGRAFÍA}

nes de escala de las distintas regiones. De modo que, según el caso, ciertas zonas podían ser dibujadas desproporcionadamente para dar relevancia a las mismas; esto sucedía a menudo con la representación de ciudades como Roma o Jerusalén. En algunos casos, enteras regiones como el África menor o Asia se representaban desproporcionadamente para poder incluir los detalles y anotaciones requeridos. Lo mismo ocurre con las relaciones de distancia: era sumamente común, por ejemplo, ubicar a Jerusalén al centro del mundo. Todo esto nos da una interesante idea de la cultura de la época, pero también de las circunstancias de producción de tal o cual mapa. Las correctas dimensiones y escalas en términos cartesianos, a las que hoy en día estamos acostumbrados, en esta época claramente están ausentes. Por su lado, las relaciones de tamaño, distancia y localización del territorio, muchas veces, y de manera muy clara, responden al contexto cultural, ideológico o hasta político del realizador y de su circunstancia.

Si bien las primeras muestras de cartografía medieval eran puramente esquemáticas y se presentaban como acotaciones gráficas a un determinado texto (de valor semántico no muy distinto del de la miniatura), con el tiempo fueron transformándose y complejizándose. De nuevo, esto es evidente a partir de la clasificación propuesta por David Woodward, que se basa en cuatro tipos básicos según la forma de la representación: la tripartita, que representa los tres continentes conocidos rodeados por el océano y divididos por los cuerpos de agua antes mencionados; la cuadripartita, que a las zonas anteriores agrega en el hemisferio sur el continente de los antípodas; la zonal, que describe las zonas climáticas de la esfera terrestre, ${ }^{2}$ y la que él llama "de transición”, que es más tardía en su aparición y más compleja pues comienza a dar cuenta de los descubrimientos geográficos realizados hacia finales de la Edad Media.

Otro aspecto a comentar de los mappaemundi es su indisoluble relación con el texto escrito. Según Sergio Octavio Torres ésta será la característica más determinante de dicho tipo de cartografías que, aun cuando se logren independizar del codex, se volverán ellas mismas texto. Él mismo hace notar que los principales corpora de mappaemundi medievales son llamados isidorianos, orosianos y macrobianos, no con base en los autores que los realizaron, sino en los autores de los textos en que estaban incluidos. Así pues, los primeros mapas registrados del Bajo Medioevo estaban incluidos, al igual que cualquier miniatura, al margen de algún texto con el fin de ilustrar, de manera muy esquemática, el contenido de éste. A este propósito, Woodward afirma que: "In the Middle Ages, the word (especially the oral word) was predominant over the image and was prescribed as such by the nature of the biblical narrative and the views of the early church fathers. Saint Gregory the Great stated that pictures were for the illiterate what the Scriptures were for those who could read" (1987: 286). Esto nos conduce también a pensar en las fuentes con cuya base se realizaban los distintos mappaemundi, las

\footnotetext{
${ }^{2}$ Algunas hipótesis de la época sostenían que los dos polos de la tierra debían ser sumamente fríos y el Ecuador sumamente caliente, por lo cual ninguna de estas zonas era apta para la vida, sólo las zonas templadas podían sostener la vida y estaban decididamente incomunicadas por la línea ecuatorial.
} 
cuales eran decididamente textuales de origen clásico o bíblico, es decir, aquéllas de reconocida autoridad. Un dato interesante a resaltar es que en este tipo de mapas las literaturas de viajes no comienzan a ser fuentes relevantes, sino de manera más bien tardía. Al respecto, Woodward comenta:

It is in the map made by Fra Mauro in 1459 that the greatest influence of the Marco Polo narratives is seen before the printed editions of them began to be disseminated. This map stands at the culmination of the age of medieval cartography [...]. It is transitional in the sense that it included information derived from portolan charts, from Ptolemy's Geography, and from the new discoveries in Asia (1987: 315).

Por lo tanto, los mappaemundi son usados para ilustrar y transmitir contenidos más bien relacionados con la historia y con la fe:

Sin el mapamundi diría que es difícil, si no imposible, imaginar o concebir en la mente esos pasajes que hablan de los hijos o nietos de Noé y de las cuatro monarquías y de otros reinos y provincias tanto en textos religiosos como profanos [...] el texto sin la ayuda de imágenes no limita los bordes de las provincias que se extienden bajo las varias partes del cielo de forma tan clara como cuando son vistas por el ojo (Paolino Minorita, en Sáenz-López, 2014: 41).

Su finalidad didáctica es evidente; por ejemplo, cuando comienzan a ser elaborados y utilizados como material didáctico en las universidades, en donde la cartografía se relaciona directamente con tres de las cuatro materias del cuadrivio, es decir, con la aritmética, la astronomía y la geometría: "The place of man in the terrestrial, celestial, and spiritual world was a central concern for medieval philosophers, and such geographical issues as the nature, shape, and size of the earth were of perennial interest" (Woodward, 1987: 306).

Por otro lado, para Torres el valor didáctico del mapa se vuelve cada vez más relevante a la vez que éste se va independizando del codex para convertirse en "objeto particular". Mientras que la cartografía comienza a depender menos de los contenidos del texto que acompaña y a reclamar sus propios contenidos y medios de significación, es más fácil separarla del libro; y el paso decisivo parece ser la inclusión de mapas en la arquitectura y la decoración, en forma de murales, o tapices, o formas escultóricas. Estos mapas, que Torres llama "de inscripción", dan cuenta de la cultura enciclopedista al buscar concentrar variados tipos de información relevante para la cultura de la época, misma que al ser sacada del scriptorium democratiza el acceso a los contenidos ahí vertidos.

Un último aspecto sobre las características de los mappaemundi medievales que es interesante destacar tiene que ver con la relación espacio-temporal que en éstos se establece. En la actualidad el mapa es concebido tan sólo como una representación del espacio, y si hay una relación temporal implicada, como es el caso de los mapas históricos, el tiempo ahí representado suele ser fijo y específicamente determinado. En 


\section{$22 \square$ LA REPRESENTACIÓN DEL MUNDO EN LA EDAD MEDIA: LA CARTOGRAFÍA}

el mappamundi medieval, en cambio, era posible representar también una narrativa, más o menos a la manera en que ocurría en aquellas pinturas que representaban una escena reproduciendo a sus personajes en distintas actitudes dentro del mismo cuadro. Así, en estos mappaemundi podían encontrarse, por ejemplo, representaciones de momentos importantes de la fe pertenecientes al Antiguo y al Nuevo Testamento, pero también a obras de santos más actuales, sin una relación cronológica claramente sistematizada. Un ejemplo de ello son los mapas incluidos en el comentario al Apocalipsis del Beato de Liébana del siglo VII: "The Beatus maps illustrated the Fall and the transforming of the Earth into the Paradise of the universal church, awaiting the moment of Revelation, where the Antichrist would be defeated and God's permanent kingdom would be established" (Woodward, 1987: 13).

Woodward denomina a buena parte de la cartografía producida en los siglos XIV y XV como "de transición", pues en ella nota la atención a modelos geográficos redescubiertos (en particular la Geografía de Claudio Ptolomeo traducida al latín a inicios del siglo XV) y a las literaturas de viajes, como el texto de Marco Polo, que daban cuenta de un mundo más extenso del conocido hasta algunos siglos atrás. Para el autor estos mapas anuncian de algún modo los modos de la cartografía del Renacimiento. No obstante, para Torres los mappaemundi medievales (que en algunos casos se seguirán reproduciendo incluso hasta el siglo XVII) permanecerán siendo decididamente medievales por su fuerte lazo con el texto y con la doctrina por encima de un sentido útil y práctico de ubicación en el espacio.

\section{Consideraciones finales}

Las teorizaciones en torno al concepto de espacio realizadas por la geografía de finales del siglo XX han sido, por algunos, leídas como una revolución en la manera de leer el mundo. Lo anterior en virtud de que en los últimos años dicho concepto se ha ido desembarazando de ciertas cualidades absolutas, que poco a poco habían ido adquiriendo a raíz de su última revolución, en el Renacimiento, cuando una serie de eventos modificaron también la manera de concebir el mundo. Muchas son las circunstancias que guiaron la transición de la imago mundi de la medieval a la renacentista, pero dos suelen ser las más referidas: la primera, de tipo más bien práctico, la navegación de exploración (que dio paso a descubrimientos y a expediciones de colonización); y la segunda, de tipo teórico, la invención de la perspectiva visual.

De manera breve hay que recordar que uno de los efectos de la invención de la perspectiva consiste en la codificación de la mirada al presuponer un espectador inmóvil que mire desde un punto fijo a un espacio que se ofrece como inmutable. Posteriormente, con Descartes el espacio "real", el habitado por los hombres, va a ser matemáticamente codificado y con ello terminará considerándosele igualmente inmutable y por ende medible y transitable. En fin, ese nuevo mundo que poco a poco se va descubriendo surcando los océanos, va a ser trazado, delimitado y representado de una 
manera mucho más precisa y con intenciones mucho más funcionales que como ocurría en la época anterior.

La cartografía medieval leída desde la geografía lefevriana puede suscitar interesantes reflexiones en torno al papel de las relaciones de poder y de los aspectos de la cultura dentro de la concepción y la construcción del espacio. En fin, lo que señalan las propuestas de la nueva geografía es que las formas de representar el espacio dicen más sobre la cultura que lo representa que sobre el espacio en sí. Esta afirmación encaja muy bien en el análisis de la cartografía medieval. En tal sentido quizá sea el caso de preguntarnos también qué es lo que dicen de nosotros las nuevas formas de hacer cartografía que se caracterizan, en cierta medida, por contestar los mapas cartesianos y por proponer "nuevas" formas discursivas (y muchas veces multidiscursivas) de concebir el espacio y el mundo.

\section{Obras citadas}

Alighieri, Dante. 2016. Questio de Aqua et Terra. Ed. y trad. Julia BenAVEnT. Madrid: Fundación Aquae.

Benavent, Julia. 2016. “A modo de introducción”. Dante Alighieri, Questio de Aqua et Terra. Madrid: Fundación Aquae, 2016. Pp. 17-35.

Cosgrove, Denis. 2005. "Mapping/Cartography”. Cultural Geography. A Critical Dictionary of Key Concepts. Ed. David AtKInson et al. Londres: I. B. Tauris. Pp. 27-33.

DuczA, Matthew. 2013. "Medieval world maps. Diagrams of a Christian Universe". University of Melbourne Collections, junio. Pp. 8-14.

HARVEY, P. D. A. 1987a. "Local and Regional Cartography in Medieval Europe". The History of Cartography. Cartography in Prehistoric, Ancient, and Medieval Europe and the Mediterranean, vol. 1. Ed. P. D. A. HARVEY y David Woodward. Chicago: University of Chicago Press. Pp. 464-500.

1987b. "Medieval Maps: an Introduction". The History of Cartography. Cartography in Prehistoric, Ancient, and Medieval Europe and the Mediterranean, vol. 1. Ed. P. D. A. HARVEY y David WoodwARD. Chicago: University of Chicago Press. Pp. 283-285.

HubBard, Phil. 2005. "Space/Place”. Cultural Geography. A Critical Dictionary of Key Concepts. Ed. David ATKInson et al. Londres: I. B. Tauris. Pp. 41-48.

LefEVRE, Henri. 2013. La producción del espacio. Trad. Emilio MarTíneZ GuTIÉRREZ. Madrid: Capitan Swing.

SÁENZ-LÓPEZ PÉREZ, Sandra. 2014. "El mundo en la Edad Media a través de sus mapas: ciencia, historia y religión”. El Románico y sus mundos imaginados. Aguilar de Campo: Centro de Estudios del Románico. Pp. 10-45. 
SöDERSTRÖM, Olaf. 2005. "Representation". Cultural Geography. A Critical Dictionary of Key Concepts. Ed. David AtKInSON et al. Londres: I. B. Tauris. Pp. 11-15.

TALLY JR., Robert T. 2013. Spatiality. Nueva York: Routledge.

TORRES AgUILAR, Sergio Octavio. 2013. Relaciones entre el códice y la cartografia medieval. La influencia del texto en los mappaemundi. Universidad Complutense de Madrid. Madrid. (Tesis de Maestria.)

WoODWARD, David. 1987. "Medieval Mappaemundi". The History of Cartography. Cartography in Prehistoric, Ancient, and Medieval Europe and the Mediterranean, vol. 1. Ed. P. D. A. HARVEY y David WoOdWARD. Chicago: University of Chicago Press. Pp. 286-370. 